\title{
Prognostic value of fibrinogen-to-albumin ratio in predicting 1-year clinical progression in patients with non-ST elevation acute coronary syndrome undergoing percutaneous coronary intervention
}

\author{
DONGXU HE, YUNDI JIAO, TONGTONG YU, JIA SONG, ZONGYU WEN, JIAKE WU, \\ WEILI DUAN, NA SUN, ZHIJUN SUN and ZHAOQING SUN \\ Department of Cardiology, Shengjing Hospital of China Medical University, Shenyang, Liaoning 110022, P.R. China
}

Received October 19, 2018; Accepted June 6, 2019

DOI: $10.3892 / \mathrm{etm} .2019 .7890$

\begin{abstract}
Previous studies have indicated that fibrinogen and low serum albumin levels are associated with poor cardiovascular outcomes. The objective of the present study was to examine whether the fibrinogen-to-albumin ratio (FAR) was able to predict the 1-year prognosis of patients with non-ST elevation acute coronary syndrome (NSTE-ACS) following percutaneous coronary intervention (PCI). A total of 1,352 patients with NSTE-ACS undergoing PCI were included in this prospective study and were divided into a low-FAR group (FAR $\leq 8.713, \mathrm{n}=901)$ and a high-FAR group $(\mathrm{FAR}>8.713, \mathrm{n}=451)$. FAR was defined as the concentration ratio of fibrinogen $(\mathrm{mg} / \mathrm{dl})$ to albumin $(\mathrm{mg} / \mathrm{dl})$ multiplied by 100 . The endpoint was the incidence of major adverse cardiovascular events (MACEs), including all-cause mortality, cardiac mortality, non-fatal myocardial reinfarction and unscheduled repeat revascularisation. The predictive performance was validated by receiver-operator characteristic (ROC) curve analysis. A total of 127 MACEs were noted during the 1-year follow-up period. Multivariate Cox analysis suggested that a high FAR was an independent predictor of all-cause mortality (hazard ratio $=2.223$, 95\% confidence interval: $1.002-4.931, \mathrm{P}=0.049$ ). Regarding the predictor of MACEs, the FAR exhibited an area under the ROC curve of 0.676 with a sensitivity of 0.630 and a specificity of 0.726 . The cut-off value was 9.114 . The FAR was an independent prognostic factor in NSTE-ACS. The present results suggest that the FAR may serve as a potential prognostic indicator for patients with NSTE-ACS undergoing
\end{abstract}

Correspondence to: Professor Zhaoqing Sun, Department of Cardiology, Shengjing Hospital of China Medical University, 39 Hua Xiang Road, Tiexi, Shenyang, Liaoning 110022, P.R. China E-mail: sunzhaoqing@vip.163.com

Key words: fibrinogen-to-albumin ratio, non-ST elevation acute coronary syndrome, percutaneous coronary intervention, major adverse cardiovascular events
PCI (approval no. NCT02667548; January 29, 2016; Shengjing Hospital of China Medical University).

\section{Introduction}

Cardiovascular disease (CVD) represents a major health burden in China and throughout the world (1). Acute coronary syndrome (ACS) is the acute manifestation of ischemic heart disease, including ST-segment elevation myocardial infarction (STEMI), non-STEMI and unstable angina pectoris. ACS exhibits severe manifestations in Chinese populations and is associated with an estimated in-hospital mortality of 5\% (2). Although percutaneous coronary intervention (PCI) is now widely available, certain ACS patients have a significant risk for the development of adverse cardiac events, even after PCI. Therefore, particular focus has been directed on the identification of novel biomarkers that may be used to predict the risk of future cardiovascular events, and aid the implementation of appropriate medical strategies for patients with ACS that may eventually improve disease prognosis. ACS is characterized by unstable atherosclerotic lesions. Inflammation has an important role in the progression of coronary atherosclerosis, plaque rupture and the incidence of thrombosis (3). CVD are accompanied by an increase in several inflammatory factors, including C-reactive protein, fibrinogen, white blood cell count, ferritin and ceruloplasmin, and by a decrease in the levels of specific proteins, including albumin, transferrin and anti-thrombin (4). Previous studies have confirmed that single inflammatory markers, including C-reactive protein (5), neutrophils (6), platelets (7), fibrinogen (8) and albumin (9), are associated with the prognosis of ACS, whereas the ratio of several single markers, e.g. the neutrophil-to-lymphocyte ratio (10), may also be associated with disease prognosis. The prognostic value of the fibrinogen-to-albumin ratio (FAR) has been studied in certain types of cancer, including esophageal and breast cancer $(11,12)$. However, to date, the association of the parameter FAR with the clinical outcome of patients with non-ST elevation (NSTE)-ACS has not been investigated (13). The purpose of the present study was to explore the association between 1-year disease outcomes and FAR levels of patients admitted with NSTE-ACS undergoing PCI. 


\section{Materials and methods}

Study design and setting. The design of the present study is illustrated in Fig. 1. The study was based on a prospective observational cohort. A total of 1,352 consecutive NSTE-ACS patients (age range, 30-88 years; mean age, $62.5 \pm 10.6$ years; male, 431, female, 921) were included in the cohort, who were recruited from the 1st of January 2015 to the 31st of December 2016. The patients were hospitalized and underwent PCI at the Shengjing Hospital of China Medical University (Shenyang, China), located in Northeastern China. Among these patients, 632 consecutive cases were diagnosed with non-STEMI and 720 were diagnosed with unstable angina pectoris. The clinical and procedural data were gathered from electronic medical records, image archives and communication systems of the interventional imaging data, and the surgical history of the PCI cases. The venous blood samples were obtained within $24 \mathrm{~h}$ of admission at room temperature, and the samples were used for analysis within $2 \mathrm{~h}$ of blood collection. The levels of albumin and fibrinogen were measured in the clinical laboratory of the Shengjing Hospital (Shenyang, China). Procedural data were extracted from the surgical history of patients who underwent PCI. The angiographic parameters were estimated visually and/or with the Picture Archiving and Communication Systems of the interventional imaging data (PHILIPS UNIQ FD10; http://www.philips.com.cn/, Neusoft PACS/RIS; http://www.neusoft.com/cn?from=singlemessage). Patients were followed up at 1, 3, 6 and 12 months after discharge. The clinical follow-up data were obtained from an outpatient clinic visit or telephone interviews with the patient's general practitioner/cardiologist, the patients themselves or their family. All enrolled patients were followed up for one year after discharge. All events were adjudicated and classified by two cardiologists. The present study complied with the Declaration of Helsinki, and was granted approval by the Shengjing Hospital of the China Medical University Research Ethics Committee (Shenyang, China). Written informed consent was formally obtained from all participants.

Participants and procedures. In the present study, a total of 1,448 patients with non-STEMI and unstable angina were recruited. Patients taking corticosteroids or cytotoxic drugs, patients receiving thrombolytic therapy or heparin and patients with autoimmune diseases, hypohepatia or renal failure, cancer, hematological disorders, inflammatory or infectious diseases, pregnancy, surgery, trauma or hemorrhagic events, who were admitted within the previous 6 months of the initiation of the study, were excluded. The patients who were not treated with PCI and were not followed up for blood sample analysis were also excluded. As the use of heparin or other drugs on admission in STEMI patients may have an impact on the results of the study, the STEMI population was not included in the study, and at the same time, the patients treated with coronary artery bypass grafting (CABG) or those with prior CABG and patients unsuitable for revascularization were not included in the study. The final cohort consisted of 1,352 patients. FAR was defined as the concentration ratio of fibrinogen $(\mathrm{mg} / \mathrm{dl})$ to albumin (mg/dl) multiplied by 100 . FAR levels were stratified by tertiles [first tertile, $<7.106(n=450)$; second tertile, 7.106-8.713 $(n=451)$; third tertile, $>8.713(n=451)]$. A high FAR $(\mathrm{n}=451)$ was defined as a value in the third tertile (8.713) and a low FAR $(n=901)$ was defined as a value in the lower two tertiles $(\leq 8.713)$.

Definitions. Unstable angina was defined as the presence of chest pain originating from cardiac dysfunction that was deteriorating (i.e., more severe, prolonged or frequent than previous episodes of angina) or occurring at rest, without serological evidence of myocyte necrosis (i.e., no elevation of the serum concentration of troponin or of the levels of the MB isoenzyme of creatine kinase). Non-STEMI was defined as the presence of cardiac chest pain with serological evidence of myonecrosis in the absence of ST-segment elevation.

Clinical end-points. The following endpoints were recorded: All-cause mortality, cardiac mortality, non-fatal myocardial reinfarction and unscheduled repeat revascularisation, which included the incidence of unscheduled repeat PCI and/or surgical bypass of target or non-target vessels.

Statistical analysis. Quantitative variables were expressed as the mean \pm standard deviation or median (interquartile range) and compared with an independent-samples t-test or the Mann-Whitney U-test. Categorical variables were represented as counts and proportions (\%) and compared with a chi-squared test. The Cox proportional-hazard regression model was used to analyze the effects of variables on event-free survival. Kaplan-Meier survival curves were further constructed to assess the prognostic value of the FAR. Multivariate Cox analysis was used for the significant variables identified in the univariate analysis. These variables included age, sex, history of diabetes mellitus, history of hypertension, heart rate on admission, left ventricular ejection fraction (LVEF), leukocyte count, neutrophil count, platelet count, troponin-I on admission, creatinine, high-density lipoprotein (HDL), fibrinogen-to-albumin ratio, left main disease, three-vessel disease, and beta-blockers. The results are reported as hazard ratios (HRs) with the corresponding 95\% confidence intervals (CIs). The accuracy of FAR for the prediction of major adverse cardiovascular events (MACEs) was assessed according to the area under the receiver-operating characteristic curve (ROC-AUC) with the MedCalc software for Windows version 11.4.2.0 (MedCalc Software). All of the tests were two-sided and $\mathrm{P}<0.05$ was considered to indicate statistical significance. All statistical analyses were performed with the SPSS version 19 (IBM Corp.).

\section{Results}

Baseline characteristics. The sample consisted of 1,352 NSTE-ACS patients who underwent PCI. The major clinical, demographic and angiographic features, description of PCI and discharge medications of patients according to their FAR are described in Table I. The patients of the high FAR group were older, with a lower percentage of female subjects, a higher incidence of diabetes mellitus and hypertension and a higher percentage of non-STEMI. Furthermore, these patients exhibited a higher incidence of left main disease and three-vessel disease and a higher frequency of beta-blocker administration. They further exhibited a higher heart rate 


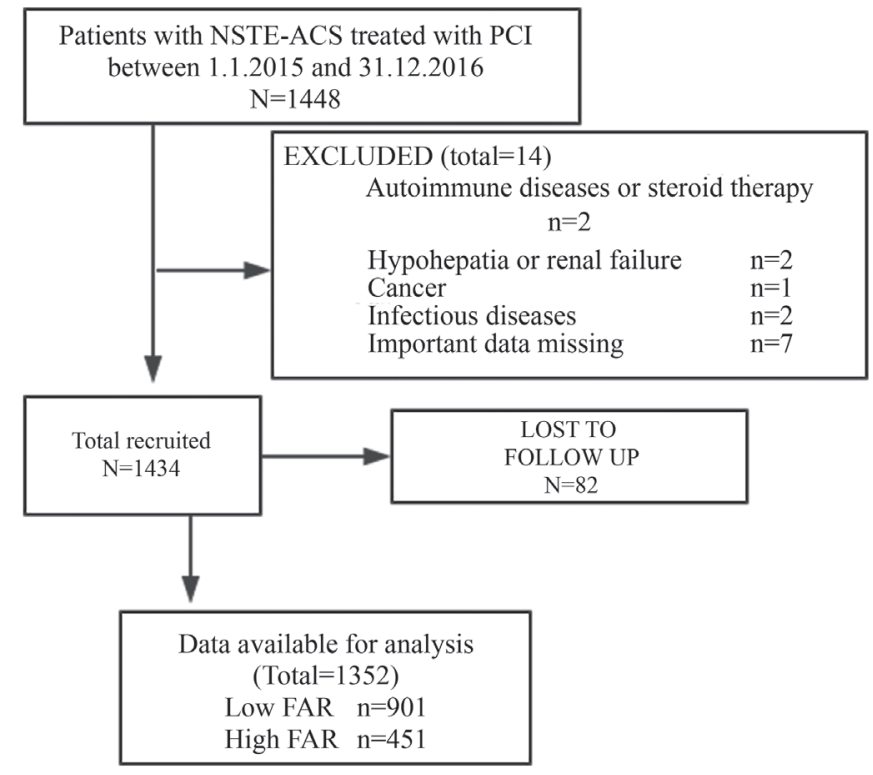

Figure 1. Flow diagram of participant selection. FAR, fibrinogen-to-albumin ratio; PCI, percutaneous coronary intervention; NSTE-ACS, non-ST elevation acute coronary syndrome.

on admission, a higher FAR and higher levels of troponin-I on admission, and elevated creatinine and fibrinogen levels. Finally, they presented with lower levels of HDL and albumin and a lower LVEF.

Clinical Outcome. The clinical outcomes at the 1-year follow-up are provided in Table II. MACEs occurred in 127 patients $(9.4 \%)$, comprising $67(14.1 \%)$ in the low FAR group and $60(13.3 \%)$ in the high FAR group. A total of 12 events of all-cause mortality, 10 events of cardiac mortality, 10 events of non-fatal myocardial reinfarction and 35 events of unscheduled repeat revascularisation were observed in the low FAR group. A total of 21 events of all-cause mortality, 17 events of cardiac mortality, 6 events of non-fatal myocardial reinfarction and 16 events of unscheduled repeat revascularisation occurred in the high FAR group. The data indicated that all-cause mortality and cardiac mortality were more frequent in the high FAR group $(\mathrm{P}<0.05)$.

Prognostic performance of different FAR levels in prediction of MACES. The results of the Cox analysis are provided in Table III. Univariate Cox analysis indicated that the FAR was an independent risk factor regarding all-cause mortality and of cardiac mortality of the NSTE-ACS patients. Multivariate Cox analysis was performed for the significant variables identified by the Univariate analysis. The data demonstrated that the FAR was an independent predictor of all-cause mortality $(\mathrm{HR}=2.223$, 95\% CI: 1.002-4.931, $\mathrm{P}=0.049)$. Kaplan-Meier curve analysis further revealed that patients with a higher FAR exhibited a higher incidence of all-cause mortality $(\mathrm{P}<0.001$; Fig. 2) and cardiac mortality $(\mathrm{P}=0.001$; Fig. 3) than those with a lower FAR (Figs. 2 and 3). The cut-off points for the sensitivity and specificity of the FAR were estimated by performing ROC curve analysis (Fig. 4). The FAR exhibited a sensitivity of 0.630 and a specificity of 0.726 for predicting MACEs (Fig. 4). The ROC-AUC was 0.676 and the cut-off value was 9.114 .

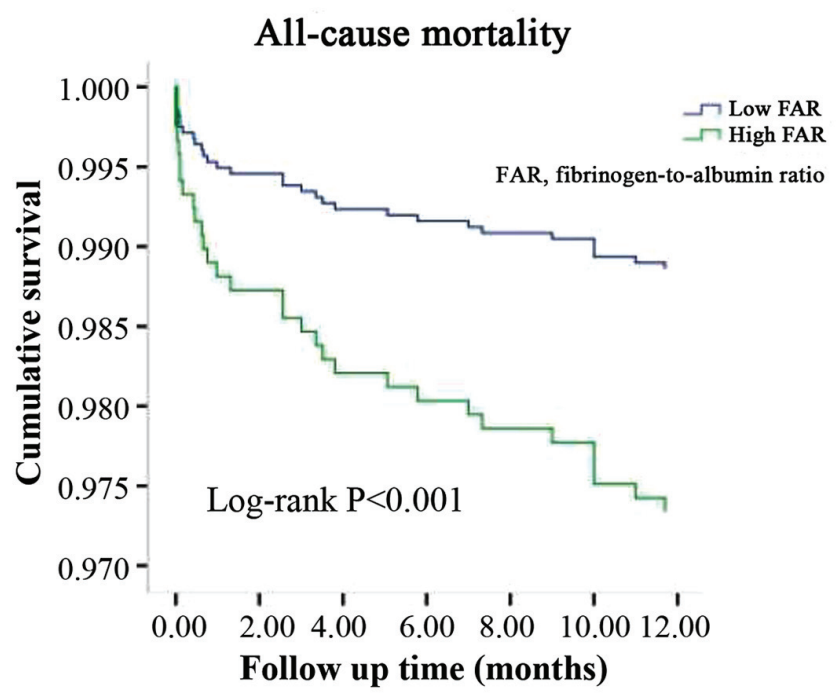

Figure 2. Kaplan-Meier survival curves of all-cause mortality based on FAR levels. FAR, fibrinogen-to-albumin ratio.

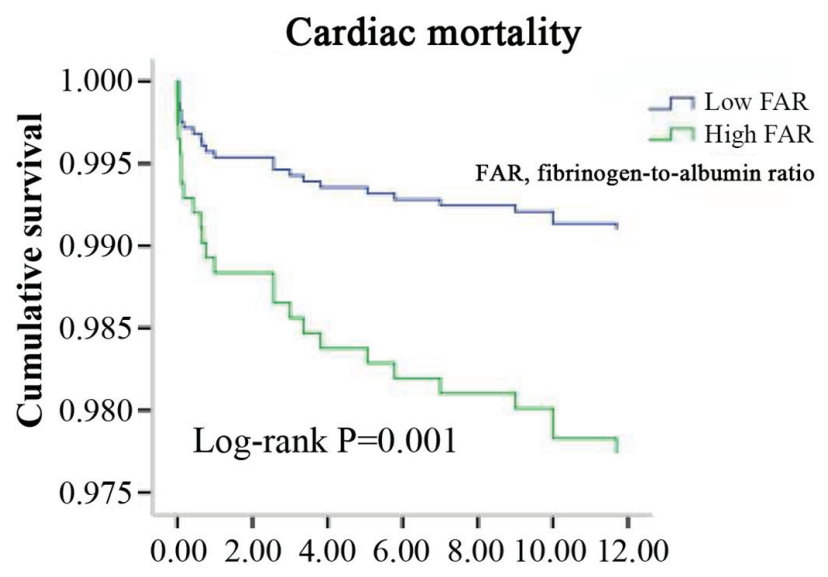

Follow up time (months)

Figure 3. Kaplan-Meier survival curves for cardiac mortality based on FAR levels. FAR, fibrinogen-to-albumin ratio.

\section{Discussion}

In the present study, a prospective analysis of 1,352 consecutive patients with NSTE-ACS treated with PCI was performed. This study found that the patients in the high FAR group were older and with a higher incidence of diabetes mellitus and hypertension. They exhibited higher levels of troponin-I on admission, elevated creatinine and a lower LVEF. These patients exhibited a higher incidence of left main disease and three-vessel disease. A previous study indicated that hypertension, diabetes, age, renal insufficiency and cardiac insufficiency are poor prognostic factors of coronary heart disease (14). Furthermore, the potential association between the FAR and MACEs in this cohort was explored. Cox analysis suggested that the FAR was independently associated with all-cause mortality in NSTE-ACS patients. The FAR exhibited an optimal accuracy for predicting MACEs, and its cut-off value in patients with NSTE-ACS undergoing PCI was 9.11 , with a sensitivity of 0.630 and a specificity of 0.726 . The 
Table I. Baseline clinical characteristics and periprocedural details.

\begin{tabular}{|c|c|c|c|}
\hline Variables & Low FAR $(n=901)$ & High FAR (n=451) & $\mathrm{P}$-value \\
\hline Age (years) & $61.5 \pm 10.4$ & $64.2 \pm 10.8$ & $<0.001^{\mathrm{a}}$ \\
\hline Female $(\%)$ & $636(70.6)$ & $285(63.2)$ & $0.006^{\mathrm{a}}$ \\
\hline Current/recent smoker & $355(39.4)$ & $164(36.4)$ & $0.279^{\mathrm{a}}$ \\
\hline Diabetes mellitus & $224(24.9)$ & $158(35.0)$ & $<0.001^{\mathrm{a}}$ \\
\hline Hypertension & $578(64.2)$ & $319(70.7)$ & $0.016^{\mathrm{a}}$ \\
\hline MI & $97(10.8)$ & $53(11.8)$ & $0.586^{\mathrm{a}}$ \\
\hline PCI & $99(11.0)$ & $60(13.3)$ & $0.213^{\mathrm{a}}$ \\
\hline Prior peripheral arterial disease & $19(2.1)$ & $11(2.4)$ & $0.697^{\mathrm{a}}$ \\
\hline $\mathrm{SBP}(\mathrm{mmHg})$ & $140.4 \pm 20.2$ & $139.6 \pm 22.5$ & $0.462^{\mathrm{c}}$ \\
\hline Heart rate (bpm) & $72(64-80)$ & $74(67-83)$ & $<0.001^{\mathrm{b}}$ \\
\hline $\operatorname{LVEF}(\%)$ & $61(58-65)$ & $61(55-64)$ & $0.010^{\mathrm{b}}$ \\
\hline Leukocyte count $\left(\mathrm{x} 10^{9} / 1\right)$ & $7.2(5.9-8.6)$ & $7.7(6.2-9.3)$ & $0.001^{\mathrm{b}}$ \\
\hline Neutrophil count $\left(\times 10^{9} / 1\right)$ & $4.7(3.8-6.5)$ & $5.2(3.9-7.5)$ & $<0.001^{\mathrm{b}}$ \\
\hline Platelet count $\left(\times 10^{9} / 1\right)$ & $201.5 \pm 58.4$ & $215.6 \pm 63.9$ & $<0.001^{\mathrm{c}}$ \\
\hline Troponin-I on admission $(\mu \mathrm{g} / \mathrm{l})$ & $0.0(0.0-0.5)$ & $0.1(0.0-1.0)$ & $<0.001^{\mathrm{b}}$ \\
\hline Creatinine $(\mu \mathrm{mol} / \mathrm{l})$ & $72.0(59.4-83.5)$ & $73.0(60.7-91.0)$ & $0.005^{\mathrm{b}}$ \\
\hline Total cholesterol (mmol/l) & $4.5 \pm 1.2$ & $4.4 \pm 1.1$ & $0.167^{\mathrm{c}}$ \\
\hline $\operatorname{HDL}(\mathrm{mmol} / \mathrm{l})$ & $1.0(0.8-1.2)$ & $0.9(0.8-1.2)$ & $0.020^{\mathrm{b}}$ \\
\hline LDL (mmol/l) & $2.8 \pm 1.0$ & $2.8 \pm 1.0$ & $0.531^{\mathrm{c}}$ \\
\hline Triglycerides (mmol/l) & $1.6(1.0-2.4)$ & $1.5(1.0-2.1)$ & $0.072^{\mathrm{b}}$ \\
\hline Fibrinogen $(g / l)$ & $2.9 \pm 0.5$ & $4.1 \pm 0.6$ & $<0.001^{\mathrm{c}}$ \\
\hline Albumin $(\mathrm{g} / \mathrm{l})$ & $41.2 \pm 3.3$ & $37.9 \pm 3.8$ & $<0.001^{\mathrm{c}}$ \\
\hline FAR & $7.1(6.2-7.8)$ & $10.3(9.4-11.6)$ & $<0.001^{\mathrm{b}}$ \\
\hline Use of glycoprotein IIb/IIIa inhibitor & $196(21.8)$ & $83(18.4)$ & $0.151^{\mathrm{a}}$ \\
\hline \multicolumn{4}{|l|}{ Diagnosis on admission } \\
\hline Unstable Angina & $524(58.2)$ & $196(43.5)$ & $<0.001^{\mathrm{a}}$ \\
\hline NSTEMI & $377(41.8)$ & $255(56.5)$ & \\
\hline \multicolumn{4}{|l|}{ PCI details } \\
\hline Left main disease & $74(8.2)$ & $53(11.8)$ & $0.035^{\mathrm{a}}$ \\
\hline Three-vessel disease & $185(20.5)$ & $122(27.1)$ & $0.007^{\mathrm{a}}$ \\
\hline Intra-aortic balloon pump & $4(0.4)$ & $5(1.1)$ & $0.156^{\mathrm{a}}$ \\
\hline TIMI flow grade 3 post PCI & $900(99.9)$ & $451(100.0)$ & $0.479^{\mathrm{a}}$ \\
\hline Aspirin & $895(99.3)$ & $448(99.3)$ & $0.999^{\mathrm{a}}$ \\
\hline Clopidogrel & $768(85.2)$ & $384(85.1)$ & $0.963^{\mathrm{a}}$ \\
\hline Ticagrelor & $124(13.8)$ & $62(13.7)$ & $0.994^{\mathrm{a}}$ \\
\hline Statin & $885(98.2)$ & $441(97.8)$ & $0.577^{\mathrm{a}}$ \\
\hline ACEI/ARBs & $518(57.5)$ & $282(62.5)$ & $0.076^{\mathrm{a}}$ \\
\hline Beta-blockers & $478(53.1)$ & $266(59.0)$ & $0.039^{\mathrm{a}}$ \\
\hline
\end{tabular}

Values are expressed as median (interquartile range), $\mathrm{n}(\%)$, or mean \pm standard deviation. ${ }^{\text {P }}$-values were calculated using Chi-square test. ${ }^{b} \mathrm{P}$-values were calculated using Mann-Whitney U-test. ${ }^{\mathrm{C}} \mathrm{P}$-values were calculated using independent-samples t-test. MI, myocardial infarction; bpm, beats per min; H/LDL, high/low-density lipoprotein; LVEF, left ventricular ejection fraction; PCI, percutaneous coronary intervention; NSTEMI, non-ST-segment elevation myocardial infarction; ACEI/ARBs, angiotensin-converting enzyme inhibitors/angiotensin receptor blockers; TIMI, thrombolysis in myocardial infarction; FAR, fibrinogen-to-albumin ratio.

results indicated that the FAR was an independent predictor of the 1-year clinical prognosis of NSTE-ACS patients. To date, a limited number of studies have been performed with regard to the association of the FAR with the incidence of CVD. A recent study reported that the FAR may be used as a biomarker of infarction to evaluate the severity of coronary artery disease in patients with STEMI (15). The FAR exhibited applicability in a similar manner to that for the outcome-differentiator SYNTAX score (15). To the best of our knowledge, the association of the FAR with the prognosis of NSTE-ACS has not been previously investigated. The present study suggested that the FAR may serve as a prognostic marker in NSTE-ACS. 
Table II. Major adverse cardiovascular events during the 1-year follow-up.

\begin{tabular}{lcrr}
\hline Outcome & Low FAR $(\mathrm{n}=901)$ & High FAR $(\mathrm{n}=451)$ & P-value \\
\hline All-cause mortality & $12(1.3)$ & $21(4.7)$ & $<0.001$ \\
Cardiac mortality & $10(1.1)$ & $17(3.8)$ & 0.001 \\
Non-fatal reinfarction & $10(1.1)$ & $6(1.3)$ & 0.724 \\
Unplanned repeat revascularization & $35(3.9)$ & $16(3.5)$ & 0.759
\end{tabular}

Values are expressed as n (\%). P-values were calculated using the Chi-square test. FAR, fibrinogen-to-albumin ratio.

Table III. Effects of the fibrinogen-to-albumin ratio (third vs. first and second tertile) on clinical outcomes according to univariate and multivariate analysis.

\begin{tabular}{|c|c|c|c|c|}
\hline \multirow[b]{2}{*}{ Variables } & \multicolumn{2}{|c|}{ Univariate analysis } & \multicolumn{2}{|c|}{ Multivariate analysis } \\
\hline & HR $(95 \%$ CI $)$ & P-value & HR $(95 \% \mathrm{CI})$ & $\mathrm{P}$-value \\
\hline All-cause mortality & $3.562(1.752,7.239)$ & $<0.001$ & $2.223(1.002,4.931)$ & 0.049 \\
\hline Cardiac mortality & $3.450(1.580,7.535)$ & 0.002 & $2.336(0.966,5.649)$ & 0.060 \\
\hline Non-fatal reinfarction & $0.913(0.505,1.650)$ & 0.763 & & \\
\hline Unplanned repeat revascularization & $1.204(0.438,3.314)$ & 0.719 & & \\
\hline
\end{tabular}

Univariate and multivariate Cox proportional hazard models were used to analyse the effects of variables on event-free survival. HR, hazard ratio; $\mathrm{CI}$, confidence interval.

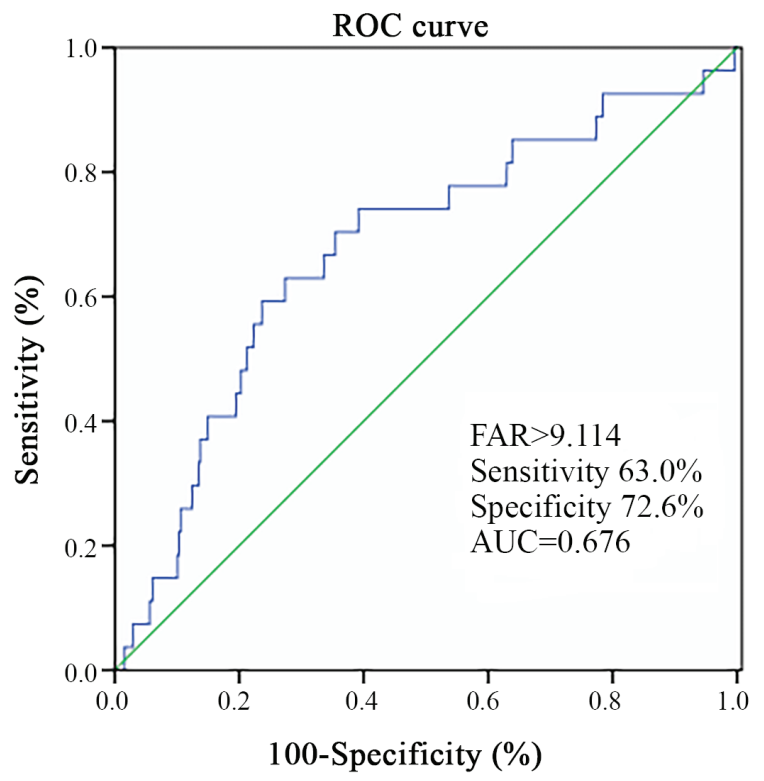

Figure 4. ROC curve indicating the predictive value of FAR. ROC, receiver operating characteristic; AUC, area under ROC curve; FAR, fibrinogento-albumin ratio.

Fibrinogen is an acute-phase protein (4). Plasma fibrinogen levels rise rapidly during the development of acute diseases, including severe bacterial infections, trauma and major surgery (8). Chronic low-grade inflammation further leads to elevated plasma fibrinogen levels, e.g. in the case of atherosclerosis $(16,17)$. Increased levels of fibrin have been confirmed in atherosclerotic lesions $(18,19)$. Fibrinogen is an important factor contributing to the coagulation process and a major component of thrombosis. In addition, this protein is considered an important inflammatory marker. High levels of fibrinogen are closely associated with the risk of CVD $(5,20,21)$. The mechanisms by which fibrinogen leads to an increase in cardiovascular risk may be explained as follows: First, fibrinogen promotes platelet aggregation. In addition, increased fibrinogen levels promote the formation of fibrin and they increase plasma viscosity $(21,22)$. Finally, fibrinogen participates in inflammatory reactions and its levels are increased in inflammatory states $(5,16,17,20)$.

Albumin is a negative acute-phase protein produced in the liver, which is associated with the prognosis of several diseases. A low serum albumin (SA) level is predictive of the clinical prognosis of patients with coronary heart disease $(9,23,24)$. Various studies have reported that hypoalbuminemia is associated with adverse outcomes, particularly in patients with CVD. This result may be explained by several mechanisms. SA exerts specific biological functions, including anti-oxidant and anti-coagulant activity, the maintenance of vascular integrity, the induction of vasodilatory effects, and the activation of toxin binding and cholesterol transport $(4,25)$. In addition, a decreased SA concentration may be associated with the development of inflammatory conditions (26-28) or inadequate nutritional intake (27).

Fibrinogen and albumin are closely associated with inflammation, and fibrinogen may modulate the inflammatory response by inducing the production of the pro-inflammatory cytokines interleukin (IL)-1, IL-6 and tumor necrosis factor (TNF)- $\alpha$ from peripheral blood mononuclear cells (8). Recently, pro-inflammatory cytokines, particularly TNF- $\alpha$ and IL-1, have been reported to be associated with the development 
of hypoalbuminemia in patients (28). Certain studies have indicated that fibrinogen and albumin are closely associated with fibrinogen levels that correlate inversely with those of SA $(29,30)$. The interaction between albumin and fibrinogen results in impaired fibrinogen activity (31), while infusion of albumin may decrease coagulation competence during major surgery (32). Since fibrinogen and albumin exhibit a positive and negative correlation with the inflammatory response, respectively, their ratio may be correlated with inflammation. Fibrinogen and albumin are two proteins present in the serum, and it is suggested that the FAR is more convenient and effective compared with the combination of the two markers (fibrinogen and albumin) measured independently, and the FAR is used in clinical applications.

The FAR may reflect information on inflammation and may be used as a valuable serological marker. Karahan et al (15) reported that FAR may be used to evaluate the severity of coronary artery disease in patients with STEMI. Recent studies have indicated that the FAR is associated with the severity of coronary stenosis in STEMI patients and that it is an independent predictor of all-cause mortality that may be used as a prognostic indicator in patients with STEMI (33). The present study suggested that the FAR is an independent predictor of all-cause mortality in patients with NSTE-ACS undergoing PCI. A limited number of studies have been performed on the prognostic value of FAR in patients with ACS. The present results should be verified in future follow-up studies with larger sample sizes.

The present study has several limitations, including the single-center design, which may have introduced selection bias. Furthermore, the present study evaluated the SA and serum fibrinogen concentration only pre-operatively, and the patient samples were tested only once prior to PCI, which did not allow for the time-dependent assessment of the changes noted regarding the clinical and biochemical end-points. In addition, C-reactive protein (CRP) a commonly studied inflammatory factor, which is involved in multiple stress responses and serves as an important factor for the prognosis of cardiovascular disease (5). CRP was not included in the current study, which is a limitation. In addition, the subjects were patients with NSTE-ACS who underwent PCI and the STEMI population was not included. Patients treated with CABG or those who received prior $\mathrm{CABG}$ or were unsuitable for revascularisation were not included in the present study. Due to limited economic circumstances of certain patients, intermediate lesions and critical lesions were not subjected to fractional flow reserve detection, did not receive any PCI treatment, conservative medical treatment or regular follow-up and the present study did not include such patients. For the selected patients, the criteria for determining a successful PCI were the achievement of a minimum stenosis diameter reduction to $<20 \%$, grade 3 thrombolysis in myocardial infarction flow assessed by angiography, angina pectoris eliminated or significantly alleviated, and improved abnormalities in the electrocardiogram. At our medical center, only $<15 \%$ of the population is subjected to intravascular ultrasound validation (34). Therefore, studies with a larger sample size are necessary in the future. Taken together, the results of the present study suggested that the FAR was able to predict the 1-year prognosis of patients with NSTE-ACS treated by PCI. Further multicenter studies are required to confirm the present results.

\section{Acknowledgements}

Not applicable.

\section{Funding}

No funding was received.

\section{Availability of data and materials}

The datasets used and/or analyzed during the present study are available from the corresponding author on reasonable request.

\section{Authors' contributions}

DXH designed the study and drafted the manuscript. YDJ and TTY participated in data analysis and grouping. JS, ZYW, JKW, WLD and NS were responsible for the patient record and follow-up. ZJS and ZQS contributed to study conception and critically revised the manuscript. The final version of the manuscript was read and approved by all the authors.

\section{Ethics approval and consent to participate}

The present study complied with the Declaration of Helsinki, and was granted approval by the Shengjing Hospital of the China Medical University Research Ethics Committee (Shenyang, China). Written informed consent was formally obtained from all participants.

\section{Patient consent for publication}

Not applicable.

\section{Competing interests}

The authors declare that they have no competing interests.

\section{References}

1. GBD 2016 DALYs and HALE Collaborators: Global, regional, and national disability-adjusted life-years (DALYs) for 333 diseases and injuries and healthy life expectancy (HALE) for 195 countries and territories, 1990-2016: A systematic analysis for the Global Burden of Disease Study 2016. Lancet 390: 1260-1344, 2017.

2. Gao R, Patel A, Gao W, Hu D, Huang D, Kong L, Qi W, Wu Y, Yang Y, Harris P, et al: Prospective observational study of acute coronary syndromes in China: Practice patterns and outcomes. Heart 94: 554-560, 2008.

3. Libby P: Inflammation in atherosclerosis. Arterioscler Thromb Vasc Biol 32: 2045-2051, 2012.

4. Ahmed MS, Jadhav AB, Hassan A and Meng QH: Acute phase reactants as novel predictors of cardiovascular disease. ISRN Inflamm 2012: 953461, 2012.

5. Emerging RiskFactors Collaboration, KaptogeS,Di AngelantonioE, Pennells L, Wood AM, White IR, Gao P, Walker M, Thompson A, Sarwar N, et al: C-reactive protein, fibrinogen, and cardiovascular disease prediction. N Engl J Med 367: 1310-1320, 2012.

6. Mayer FJ, Gruenberger D, Schillinger M, Mannhalter C, Minar E, Koppensteiner R, Arbesú I, Niessner A and Hoke M: Prognostic value of neutrophils in patients with asymptomatic carotid artery disease. Atherosclerosis 231: 274-280, 2013.

7. Iijima R, Ndrepepa G, Mehilli J, Bruskina O, Schulz S, Schömig A and Kastrati A: Relationship between platelet count and 30-day clinical outcomes after percutaneous coronary interventions. Pooled analysis of four ISAR trials. Thromb Haemost 98: 852-857, 2007. 
8. Jensen T, Kierulf P, Sandset PM, Klingenberg O, Joø GB, Godal HC and Skjønsberg OH: Fibrinogen and fibrin induce synthesis of proinflammatory cytokines from isolated peripheral blood mononuclear cells. Thromb Haemost 97: 822-829, 2007.

9. Kurtul A, Murat SN, Yarlioglues M, Duran M, Ocek AH Koseoglu C, Celık IE, Kilic A and Aksoy O: Usefulness of serum albumin concentration to predict high coronary SYNTAX score and in-hospital mortality in patients with acute coronary syndrome. Angiology 67: 34-40, 2016.

10. Duffy BK, Gurm HS, Rajagopal V, Gupta R, Ellis SG and Bhatt DL: Usefulness of an elevated neutrophil to lymphocyte ratio in predicting long-term mortality after percutaneous coronary intervention. Am J Cardiol 97: 993-996, 2006.

11. Tan Z, Zhang M, Han Q, Wen J, Luo K, Lin P, Zhang L, Yang H and Fu J: A novel blood tool of cancer prognosis in esophareal squamous cell carcinoma: The Fibrinogen/Albumin Ratio. J Cancer 8: 1025-1029, 2017.

12. Hwang KT, Chung JK, Roh EY, Kim J, Oh S, Kim YA, Rhu J and Kim S: Prognostic influence of preoperative fibrinogen to albumin ratio for breast cancer. J Breast Cancer 20: 254-263, 2017.

13. Chan D, Lawson L and Kunadian V: Management of older patients presenting with non-ST-elevation acute coronary syndrome. EuroIntervention 14: e258-e260, 2018.

14. 2012 Writing Committee Members, Jneid H, Anderson JL, Wright RS Adams CD, Bridges CR, Casey DE Jr, Ettinger SM, Fesmire FM, Ganiats TG, etal:2012 ACCF/AHA focused update of the guideline for the management of patients with unstable anginan/Non-ST-elevation myocardial infarction (updating the 2007 guideline and replacing the 2011 focused update): A report of the American College of Cardiology Foundation/American Heart Association Task Force on practice guidelines. Circulation 126: 875-910, 2012.

15. Karahan O, Acet H, Ertaş F, Tezcan O, Çalişkan A, Demir M, Kaya AF, Demirtass S, Cevik MU and Yavuz C: The relationship between fibrinogen to albumin ratio and severity of coronary artery disease in patients with STEMI. Am J Emerg Med 34 1037-1042, 2016

16. Stone MC and Thorp JM: Plasma fibrinogen-a major coronary risk factor. J R Coll Gen Pract 35: 565-569, 1985.

17. Wilhelmsen L, Svärdsudd K, Korsan-Bengtsen K, Larsson B, Welin L and Tibblin G: Fibrinogen as a risk factor for stroke and myocardial infarction. N Engl J Med 311: 501-505, 1984.

18. Hoff HF, Heideman CL, Jackson RL, Bayardo RJ, Kim HS and Gotto AM Jr: Localization patterns of plasma apolipoproteins in human atherosclerotic lesions. Circ Res 37: 72-79, 1975.

19. Sadoshima S and Tanaka K: Fibrinogen and low density lipoprotein in the development of cerebral atherosclerosis. Atherosclerosis 34: 93-103, 1979.

20. Mahmud E, Behnamfar O, Lin F, Reeves R, Patel M and Ang L: Elevated serum fibrinogen is associated with 12-month major adverse cardiovascular events following percutaneous coronary intervention. J Am Coll Cardiol 67: 2556-2557, 2016.

21. Ang L, Behnamfar O, Palakodeti S, Lin F, Pourdjabbar A, Patel MP, Reeves RR and Mahmud E: Elevated baseline serum fibrinogen: Effect on 2-year major adverse cardiovascular events following percutaneous coronary intervention. J Am Heart Assoc 6, 2017.

22. Lowe GD, Fowkes FG, Dawes J, Donnan PT, Lennie SE and Housley E: Blood viscosity, fibrinogen, and activation of coagulation and leukocytes in peripheral arterial disease and the normal population in the Edinburgh Artery Study. Circulation 87: 1915-1920, 1993
23. Chien SC, Chen CY, Leu HB, Su CH, Yin WH, Tseng WK, Wu YW, Lin TH, Chang KC, Wang JH, et al: Association of low serum albumin concentration and adverse cardiovascular events in stable coronary heart disease. Int J Cardiol 241: 1-5, 2017.

24. Wada H, Dohi T, Miyauchi K, Shitara J, Endo H, Doi S, Naito R, Konishi H, Tsuboi S, Ogita M, et al: Impact of serum albumin levels on long-term outcomes in patients undergoing percutaneous coronary intervention. Heart Vessels 32: 1085-1092, 2017.

25. Roche M, Rondeau P, Singh NR, Tarnus E and Bourdon E: The antioxidant properties of serum albumin. FEBS Lett 582: 1783-1787, 2008

26. Nicholson JP, Wolmarans MR and Park GR: The role of albumin in critical illness. Br J Anaesth 85: 599-610, 2000.

27. Don BR and Kaysen G: Serum albumin: Relationship to inflammation and nutrition. Semin Dial 17: 432-437, 2004

28. Odamaki M, Kato A, Takita T, Furuhashi M, Maruyama Y, Yonemura K and Hishida A: Role of soluble receptors for tumor necrosis factor alpha in the development of hypoalbuminemia in hemodialysis patients. Am J Nephrol 22: 73-80, 2002.

29. Kim KJ, Yang WS, Kim SB, Lee SK and Park JS: Fibrinogen and fibrinolytic activity in CAPD patients with atherosclerosis and its correlation with serum albumin. Perit Dial Int 17: 157-161, 1997.

30. Kim SB, Chi HS, Park JS, Hong CD and Yang WS: Effect of increasing serum albumin on plasma D-dimer, von Willebrand factor, and platelet aggregation in CAPD patients. Am J Kidney Dis 33: 312-317, 1999.

31. Galanakis DK: Anticoagulant albumin fragments that bind to fibrinogen/fibrin: Possible implications. Semin Thromb Hemost 18: 44-52, 1992.

32. Rasmussen KC, Højskov M, Johansson PI, Kridina I, Kistorp T, Salling L, Nielsen HB, Ruhnau B, Pedersen T and Secher NH: Impact of albumin on coagulation competence and hemorrhage during major surgery: A randomized controlled trial. Medicine (Baltimore) 95: e2720, 2016.

33. Xiao L, Jia Y, Wang X and Huang H: The impact of preoperative fibrinogen-albumin ratio on mortality in patients with acute ST-segment elevation myocardial infarction undergoing primary percutaneous coronary intervention. Clin Chim Acta 493: 8-13, 2019.

34. Smith SC, Dove JT, Jacobs AK, Kennedy JW, Kereiakes D, Kern MJ, Kuntz RE, Popma JJ, Schaff HV, Williams DO, et al: ACC/AHA guidelines for percutaneous coronary intervention (revision of the 1993 PTCA guidelines)-executive summary: A report of the American College of Cardiology/American Heart Association task force on practice guidelines (Committee to revise the 1993 guidelines for percutaneous transluminal coronary angioplasty) endorsed by the Society for Cardiac Angiography and Interventions. Circulation 103: 3019-3041, 2001.

This work is licensed under a Creative Commons Attribution-NonCommercial-NoDerivatives 4.0 International (CC BY-NC-ND 4.0) License. 